\title{
Ozone Therapy in diabetes and its complications
}

\section{Silvia Menendez-Cepero(1), Olga Sonia Leon(2), Gregorio Martinez(3) and Hector Alvarez(4)}

\author{
(1)Collaborator of the National Center for Scientific Research. Havana, Cuba. \\ (2)Center for Research and Biological Evaluation, University of Havana, Cuba. \\ (3)Medinat srl Clinic. Via Fazioli 22, 60021 Camerano, Italy. \\ (4)Institute of Angiology and Vascular Surgery, Havana, Cuba.
}

\section{ABSTRACT}

\section{OOPEN ACCESS}

\section{Citation}

Menendez-Cepero S, Leon OS, Martinez $\mathrm{G}$ and Alvarez $\mathrm{H}$. Ozone Therapy in diabetes and its complications [abstract]. Proceedings of the 5Th WFOT Meeting; 2016 Nov 18-20; Mumbai, India. J Ozone Ther. 2018;2(2). doi: 10.7203/jo3t. 2.2.2018.11127

\section{Academic Editor}

Jose Baeza-Noci, School of Medicine, Valencia University, SPAIN

\section{Editor}

World Federation of Ozone Therapy, Bolgna, ITALY

\section{Received}

December 9, 2017

\section{Accepted}

December 10, 2017

\section{Published}

March 4, 2018

\section{Intellectual Property}

Silvia Menendez-Cepero. This is an open access article distributed under the terms of the Creative Commons Attribution License (CC BY 4.0), which permits unrestricted use, distribution, and reproduction in any medium, provided the original author and source are credited.
In Diabetes mellitus, long-term complications, that cause morbidity and premature mortality, are characterized by microvascular and macrovascular diseases. A more frequent concomitant of distal anesthesia is the development of neurotropic ulceration, particularly on the plantar aspect of the foot. All these events characterize the underlying mechanisms that may lead to rapid gangrene after foot injury. Vascular endothelium appears to be a vulnerable target for hyperglycemia-induced metabolic changes. Activation of polyol pathway, non-enzymatic glycosilation of proteins and the increase of reactive oxygen species (ROS) play an important role in diabetes complications.

Ozone has been used as a therapeutical agent and beneficial effects have been observed. However, so far only a few biochemical and pharmacodynamic mechanisms have been elucidated. We demonstrated that controlled ozone administration may promote an oxidative preconditioning or adaptation to oxidative stress, preventing the damage induced by ROS [1]. Taking into account that diabetes is a disorder associated with oxidative stress, we postulate that ozone treatment might protect antioxidant systems and maintain, at a physiological level, other markers of endothelial cell damage associated with diabetic complications.

In this study we evaluate ozone efficacy in a preclinical diabetes animal model [2]and in a clinical trial with type 2 diabetes patients suffering of diabetic foot complications [3].

Diabetes animal model [2]: Five groups of rats were classified as follows: (1) control group treated only with physiological saline solution; (2) positive control group using streptozotocin (STZ) as a diabetes inductor; (3) ozone group, receiving 10 treatments $(1.1 \mathrm{mg} / \mathrm{kg})$, one per day after STZ-induced diabetes; (4) oxygen group (26 $\mathrm{mg} / \mathrm{kg}$ ), one per day, as in group 3 , but using oxygen only and (5) control ozone group, as in group 3 , but without STZ. The following parameters were evaluated: plasma glucose concentration; in pancreas homogenates: levels of aldose reductase, fructolysine, advanced oxidation protein products, nitrite/nitrates (as an index of nitric oxide), glutathione (GSH), total hydroperoxides (TH), malondialdehide concentration (MDA), catalase (CAT), superoxide dismutase (SOD) and glutathione peroxidase (GSH-Px) activities as indicators of redox balance. Pancreas morphology was evaluated by light microscopy.

Clinical assay [3]: Randomized controlled clinical trial where all patients provided a signed informed consent before being enrolled was performed. Adult hospitalized patients of both sex of any ethnic, with diagnosis of 
Author Information

silviamenendez@infomed.sld.cu neuroinfectious diabetes foot suffering of ulcers of the feet and lower extremities were eligible to participate in the study. These patients must not meet any of the following criteria: severe septic conditions, hypersensibility to the medication that will be used, hepatic dysfunction, renal failure (serum creatinine level $>1.32 \mathrm{~mol} / \mathrm{L}$ ), pregnancy, cancer, current therapy with any immunosuppresive agent or anticonvulsant. Patients were randomized to two different groups of treatment: 1- control, 50 patients treated with antibiotic therapy, systemic and topically in the lesion (during 20 days), and 2- ozone, 52 patients treated daily with ozone, 20 sessions, by rectal insufflation (with an ozone dose of $10 \mathrm{mg}$, ozone concentration: $40 \mathrm{mg} / \mathrm{L}$ ) and locally. The same biochemical parameters that were taking into account in the preclinical study were measured in plasma, at the beginning and at the end of the treatments, as well as the clinical evaluation of the lesions and length of hospitalization.

In both studies, ozone treatment improved glycemic control, and prevented oxidative stress, the increase of fructolysine content and advanced oxidation protein products. However, high plasma glucose figures and oxidative stress were maintained in the antibiotic group. In the animal model, ozone treatment also improved pancreas integrity. In the clinical assay, at the end of the treatments, a decrease of the area and perimeter of the lesions, for both groups, was obtained, but the expected total recovery showed that patients treated with ozone needed half of the time to achieve it, with regard to the antibiotic group. No significant differences between both treatments were obtained for the variable clinical evaluation (qualitative clinical evaluation), but with a trend to increase the number of cured patients and to decrease the non-cured patients in the ozone group, in comparison with also decreased the area to be amputated. The length of hospitalization decreased in patients treated with ozone with regard to the antibiotic group.

Also, a controlled, randomized and double blind clinical trial was performed in 21 patients with a diagnostic criterion of peripheral diabetic neuropathy (DN) which were treated with ozone (ozone group) or with oxygen (oxygen group), both by rectal insufflation. The results of this study showed that repeated administration of ozone in non-toxic doses played a role in the control of diabetes and its complications. It is important to emphasize the ozone treatment effect in the increase of SOD, as well as to consider it a molecular target in this syndrome. This is an interesting explanation, since it has been recognized anion superoxide radical as the link of four pathogenic pathways associated to micro and macro vascular complications in diabetes.

The ozone treatment, in patients with diabetes type 2 suffering of neuroinfectious diabetic foot, improved glycemic control and prevented oxidative stress associated to diabetes mellitus and its complications, maintaining a cellular redox balance, in agreement with the excellent results obtained clinically in these patients. Patients with DN treated with ozone showed significant statistical increases in amplitude of peroneal (proximal and distal) nerve for both legs and nerve conduction velocity, as well as improvement in numbness, paresthesia and painful dysesthesia of the feet. However, no significant modification was observed in patients treated with oxygen in any of the electrophysiological indicators or clinical symptoms measured, between the pre and post treatment values No side effects were observed.

Ozone therapy could be a future alternative in the therapy of diabetes and its complications.

Keywords: ozone therapy, diabetes, oxidative preconditioning, oxidative stress. 


\section{References:}

1. Leon OS, Menendez S, Merino N, Castillo R, Sam S, Perez L, et al. Ozone oxidative preconditioning: a protection against cellular damage by free radicals. Mediat Inflamm. 1998;7:289-294.

2. Al-Dalain M, Martinez G, Candelario-Jalil E, Menendez S, Re L, Giulani $A$, et al. Ozone treatment reduces markers of oxidative and endothelial damage in an experimental diabetes model in rats. Pharmacol Res. 2001;44(5):391-396.

3. Martinez G, Al-Dalain SM, Menendez S, Re L, Giuliani A, Candelario-Jalil $\mathrm{E}$, et al. Therapeutic efficacy of ozone medical treatments in patients with diabetic foot. Eur J Pharmacol. 2005;523:151-161.

4. Menendez S, Fernandez J, Turrent J, Colmenero M. Ozone therapy in patients with diabetic neuropathy. Rev CENIC Ciencias Biológicas. 1998;29(3):165-168. 